\title{
Automatic Event Detector from Smartphone Accelerometry: Pilot mHealth Study for Obstructive Sleep Apnea Monitoring at Home
}

\author{
Ignasi Ferrer-Lluis, Yolanda Castillo-Escario, Josep Maria Montserrat, Raimon Jané, Senior Member, IEEE
}

\begin{abstract}
Obstructive sleep apnea (OSA) is a common disorder with a low diagnosis ratio, leaving many patients undiagnosed and untreated. In the last decades, accelerometry has been found to be a feasible solution to obtain respiratory activity and a potential tool to monitor OSA. On the other hand, many smartphone-based systems have already been developed to propose solutions for OSA monitoring and treatment. The objective of this work was to develop an automatic event detector based on smartphone accelerometry and pulse oximetry, and to assess its ability to detect thoracic movements. It was validated with a commercial OSA monitoring system at home. Results of this preliminary pilot study showed that the proposed event detector for accelerometry signals is a feasible tool to detect abnormal respiratory events, such as apneas and hypopneas, and has potential to be included in smartphone-based systems for OSA assessment.
\end{abstract}

\section{INTRODUCTION}

Obstructive sleep apnea (OSA) is a sleep related disorder marked by an involuntary total or partial occlusion of the upper airways. This occlusion is responsible for a reduction of the respiratory flow that induces the appearance of periods of hypoxia. Moderate to severe OSA is estimated to affect 7$21 \%$ of the adult men and $2-11 \%$ of the adult women, specially on obese population [1]. However, one major burden is that $75-80 \%$ of the cases remain undiagnosed and untreated [2], partly due to the difficulty to reproduce home sleep environment and the cost of the OSA gold standard test: polysomnography (PSG).

Smartphones have appeared to be a suitable solution for mobile health monitoring due to their availability and characteristics. In the last years, different approaches have demonstrated the feasibility of using accelerometry to detect respiratory rate [3-4]. Moreover, smartphone-based accelerometry has begun to be used in different studies for

*Research funded by fellowships from "la Caixa" Foundation (ID 100010434, fellowship codes LCF/BQ/IN17/11620029, LCF/BQ/ ES18/11670019), the EU Horizon 2020 research and innovation program under the Marie Sklodowska-Curie grant No. 713673,CERCA Program, Secretaria d'Universitats i Recerca de la Generalitat de Catalunya (GRC 2017 SGR 01770), the Spanish Ministry of Economy and Competitiveness (DPI2015-68820-R MINECO/FEDER) and ISCIII (FIS PI17/01068).

I. Ferrer-Lluis, Y. Castillo-Escario and R. Jané are with the Institute for Bioengineering of Catalonia (IBEC), the Barcelona Institute of Science and Technology (BIST), Centro de Investigación Biomédica en Red de Bioingeniería, Biomateriales y Nanomedicina (CIBER-BBN) and ESAII Department, Universitat Politècnica de Catalunya - BarcelonaTech (UPC), Barcelona, Spain (e-mail: \{ycastillo, iferrer, rjane\} @ibecbarcelona.eu).

J. M. Montserrat is with Hospital Clínic de Barcelona (Sleep Lab, Pneumology Service) and Centro de Investigación Biomédica en Red de Enfermedades Respiratorias (CIBERES) respiratory rate assessment [5]. On the other hand, regarding OSA monitoring, multiple attempts have been performed to develop smartphone tools to assess sleep quality [6]. Among them, some did not consider accelerometry [7] and others used it just as an activity monitor [8]. Nevertheless, our group has developed some recent proof-of-concept studies which tested the feasibility of using smartphone accelerometry to characterize the respiratory activity and help monitoring OSA behavior [9].

This work is a pilot study which proposes an automatic event detector based on accelerometry and pulse oximetry to identify thoracic movement variations associated with obstructive events, such as apneas or hypopneas, and validate it with a reference commercial system for OSA diagnosis and monitoring.

\section{MATERIALS AND METHODS}

\section{A. Acquisition system}

Two independent devices have been used to record signals. The reference device was Apnealink ${ }^{\mathrm{TM}}$ Air from ResMed, which recorded air flow, via nasal cannula, thoracic effort, via chest band, and pulse oximetry, via wired finger clip. The test device was an Android 6.0.1 smartphone Samsung S5 SM-G900F coupled with a wired pulse oximeter model "Kenek-edge", ownership of LionsGate Technologies Inc. (Vancouver, Canada. Website and e-mail address: LGTmedical.com; contact@LGTmedical.com). The smartphone system recorded accelerometry and pulse oximetry and included a fastening system to hold the phone in the desired position.

\section{B. Experiment design}

The goal of this experiment is to design an automatic event detector for accelerometry in a smartphone-based system and determine event to event agreement versus a portable home sleep monitoring device. The reference device was placed over the sternum while the test device was placed above, on the chest close to the neck. Simultaneous full night recordings were performed at home. Subjects chosen were 3 males between 55-65 years old, with moderate to severe OSA, according to their apnea-hypopnea (AHI) index, estimated by the Apnealink ${ }^{\mathrm{TM}}$ device. All the experiments were approved by the Hospital Clínic Ethics Committee.

\section{Signal acquisition settings}

The reference device sampling frequencies were $100 \mathrm{~Hz}$ for air flow, $10 \mathrm{~Hz}$ for thoracic effort and $1 \mathrm{~Hz}$ for pulse oximetry. Data recording was controlled by the device and accessed through the official Apnealink ${ }^{\mathrm{TM}}$ Air software. Recordings were exported in the European data format (EDF) for further analysis. 
The test device used two sensors: the smartphone's embedded MPU-6500 triaxial accelerometer and the wired coupled pulse oximeter by LionsGate Inc. Sampling frequencies were $200 \mathrm{~Hz}$ for accelerometry signal and $2 \mathrm{~Hz}$ for pulse oximetry signal $\left(\mathrm{SpO}_{2}\right)$. To simplify signal acquisition process, data recording was managed by the Android "Automate" application and started upon phone boot. "Automate" application managed the launching of the acquisition applications for each signal acquired. Accelerometry was recorded with the third-party Android application "Sensors Logger" and data was automatically saved in smartphone's local memory in a ".txt" file. Pulse oximetry was recorded with the third-party Android application "Cooper Demo", provided by the pulse oximeter manufacturer, and data was automatically saved in smartphone's local memory in a ".csv" file. Smartphone's memory was sufficient to store full night signal recordings.

\section{Accelerometry and $\mathrm{SpO}_{2}$ signal processing and analysis: Automatic event detection}

From accelerometry, we expected to retrieve subject's sleeping position as well as thoracic movement. From pulse oximetry we expected to retrieve desaturation episodes.

Signal processing and analysis was carried out after the whole night recordings were saved. Data from each recording was loaded into R2017b Matlab ${ }^{\circledR}$ programming environment. The proposed event detection method includes the following steps:

1) Pulse oximetry signal was processed to detect desaturation episodes equal or greater than $3 \%$.

2) To retrieve subject's sleeping position, accelerometry signal was filtered with an $8^{\text {th }}$ order $0.01 \mathrm{~Hz}$ low pass Butterworth filter to obtain the baseline value produced by the gravity acceleration. Afterwards, the axis with the major contribution of the gravity was chosen as the subject's position direction. As it can be seen in Fig. 1, position options are: "Supine", "Prone", "Lateral left", "Lateral right" and "Standing". Moreover, sleep position changes were calculated by using a moving window of 6 seconds on each raw accelerometry axis, separately. For each window, position changes were detected when the higher accelerometry value minus the lower accelerometry value exceeded a fixed threshold of $1 \mathrm{~g}$. Position changes closer than 3 seconds were merged. Detected areas of position change were not considered for thoracic effort calculation.

3) To retrieve thoracic movement, an $8^{\text {th }}$ order $0.8 \mathrm{~Hz}$ low pass Butterworth filter was used to remove high frequency noise from each of the 3 channels of the raw accelerometry data. Afterwards, the angle between the accelerometry vector and the gravity vector was calculated at each sample, to merge data into a single signal. Subsequently, an $8^{\text {th }}$ order 0.1 $\mathrm{Hz}$ to $0.8 \mathrm{~Hz}$ band pass Butterworth filter was used upon the angle signal to remove the baseline and keep components associated to respiratory frequencies.

4) Once the angle signal has been filtered, the objective was to find events with a smaller angle variation, which were expected to be associated with abnormal events, such as apneas or hypopneas. To obtain those events, local maxima and minima have been calculated upon the filtered angle signal. Local maxima were used to calculate the upper envelope while local minima were used to calculate the lower envelope. Afterwards, the total amplitude of the signal was calculated by subtracting the lower envelope from the upper envelope. Then, from the total amplitude signal, two coefficients were calculated to identify amplitude reduction associated to smaller angle variation. Coefficient 1 and coefficient 2 compared each sample amplitude value to prior and subsequent total amplitude maxima, respectively. Afterwards, reductions below $70 \%$ from both coefficients were detected as respiratory events. Threshold value was chosen to mimic hypopnea flow reduction rule. Events were then processed to determine proper event onset and offset. Event detection procedure can be seen in Fig. 2.

\section{E. Reference device data validation}

Reference device events were manually validated by a sleep clinician expert, who deleted reference device's wrong automatic events, corrected mislabeled events and added missing events.

\section{F. Test device manual annotation and validation}

Test device accelerometry and $\mathrm{SpO}_{2}$ data were provided to a sleep clinician expert to proceed to blindly annotate events, according to accelerometry signal amplitude reduction with a desaturation event.

\section{G. Event to event agreement and assessment}

It has been considered that two events agree when they are totally or partially overlapped. For each comparison the number of true positives, false positives and false negatives were calculated and the sensitivity (Se \%) and positive predictive value (PPV \%) were obtained.

\section{RESULTS}

Table I shows the Se \% and PPV \% of the event to event agreement on three different comparisons: automatic detector (test) vs reference system, manual annotations (test) vs reference system and automatic detector (test) vs manual annotations (test). When comparing the total number of events from the 3 patients to the reference system, the automatic detector (test) has slightly better sensitivity $(85.22 \%$ vs $82.62 \%)$ but lower PPV values $(82.13 \%$ vs $95.27 \%$ ) than manual annotation (test) from an expert.

Table II shows the AHI estimated from the events obtained from different devices and methods. When compared to the reference system, the AHI values calculated with the proposed automatic event detector show a better AHI estimation than manual annotation.

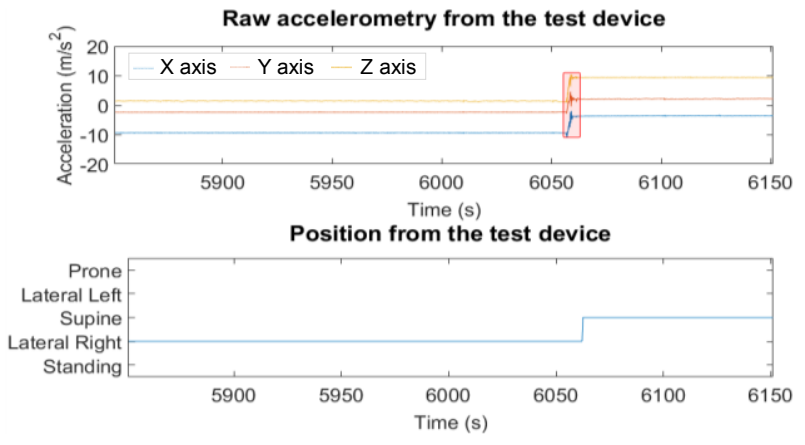

Figure 1. Triaxial accelerometry raw signal with a subject movement label (above) and the extracted position signal (below). 
TABLE I. COMPARISON OF SENSITIVITY AND POSITIVE PREDICTIVE VALUE BETWEEN TEST AND REFERENCE DEVICES

\begin{tabular}{|c|c|c|c|c|c|c|}
\hline \multirow{2}{*}{ Subject } & \multicolumn{6}{|c|}{ Event agreement } \\
\cline { 2 - 7 } & $\begin{array}{c}\text { Automatic Test }- \\
\text { Reference }\end{array}$ & \multicolumn{2}{|c|}{$\begin{array}{c}\text { Manual test }- \\
\text { Reference }\end{array}$} & \multicolumn{2}{c|}{$\begin{array}{c}\text { Automatic test }- \\
\text { Manual test }\end{array}$} \\
\cline { 2 - 7 } & Se \% & PPV \% & Se \% & PPV \% & Se \% & PPV \% \\
\hline 1 & 74.36 & 74.36 & 87.18 & 91.89 & 76.47 & 68.42 \\
\hline 2 & 89.77 & 85.41 & 80.00 & 94.59 & 95.45 & 79.25 \\
\hline 3 & 81.55 & 80.77 & 84.62 & 97.78 & 91.46 & 72.82 \\
\hline Total & 85.22 & 82.62 & 82.13 & 95.27 & 91.53 & 75.67 \\
\hline
\end{tabular}

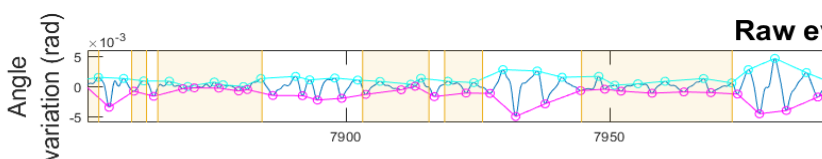

Raw event detection

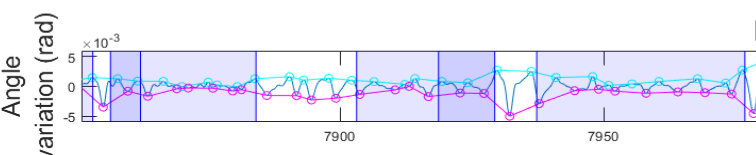

Event expansion

\begin{tabular}{|c|c|c|c|}
\hline \multirow{2}{*}{ Subject } & \multicolumn{3}{|c|}{ Apnea Hypopnea Index (AHI) } \\
\cline { 2 - 4 } & Reference device & \multicolumn{2}{|c|}{ Test device } \\
\cline { 2 - 4 } & Manual validated & Automatic events & Manual events \\
\hline 1 & 18.03 & 17.15 & 14.95 \\
\hline 2 & 34.17 & 34.37 & 25.44 \\
\hline 3 & 24.21 & 27.53 & 19.46 \\
\hline
\end{tabular}

\section{Event expansion}

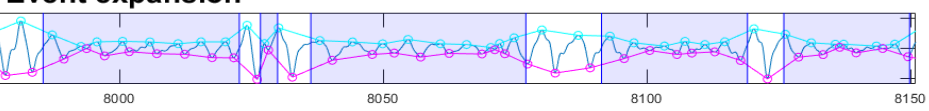

Time (s)

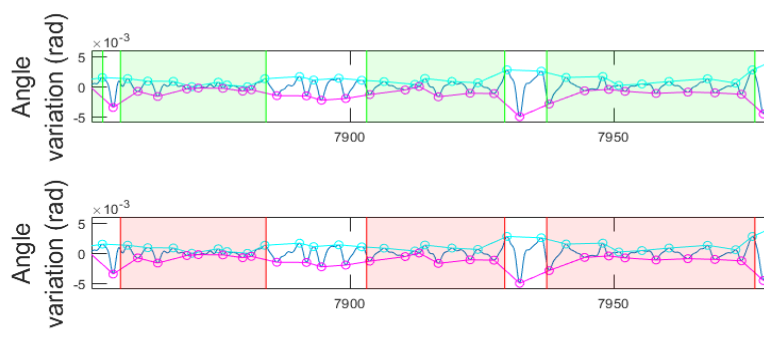

Event merging

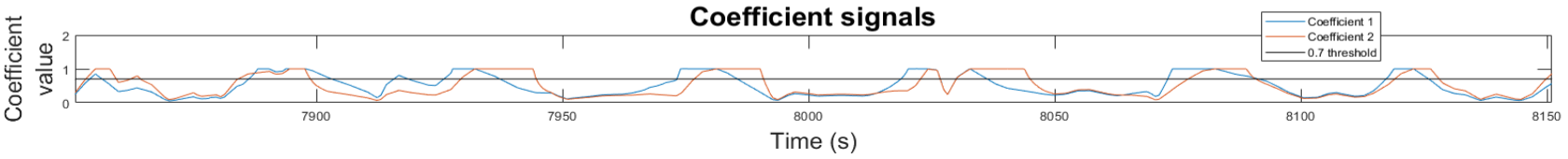

Figure 2. Accelerometry angle signal and event detection algorithm steps

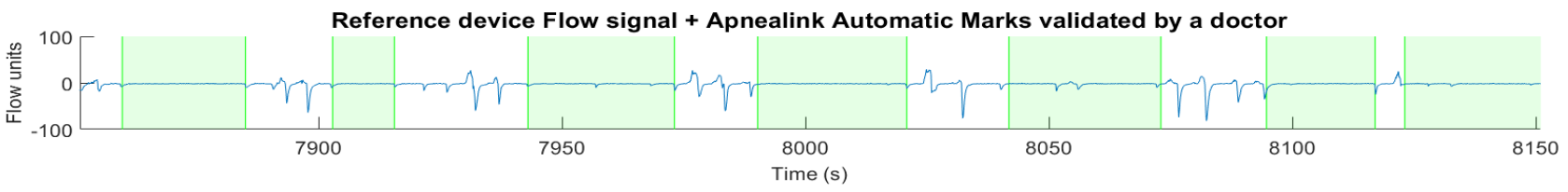

Reference device Effort signal + Apnealink Automatic Marks validated by a doctor
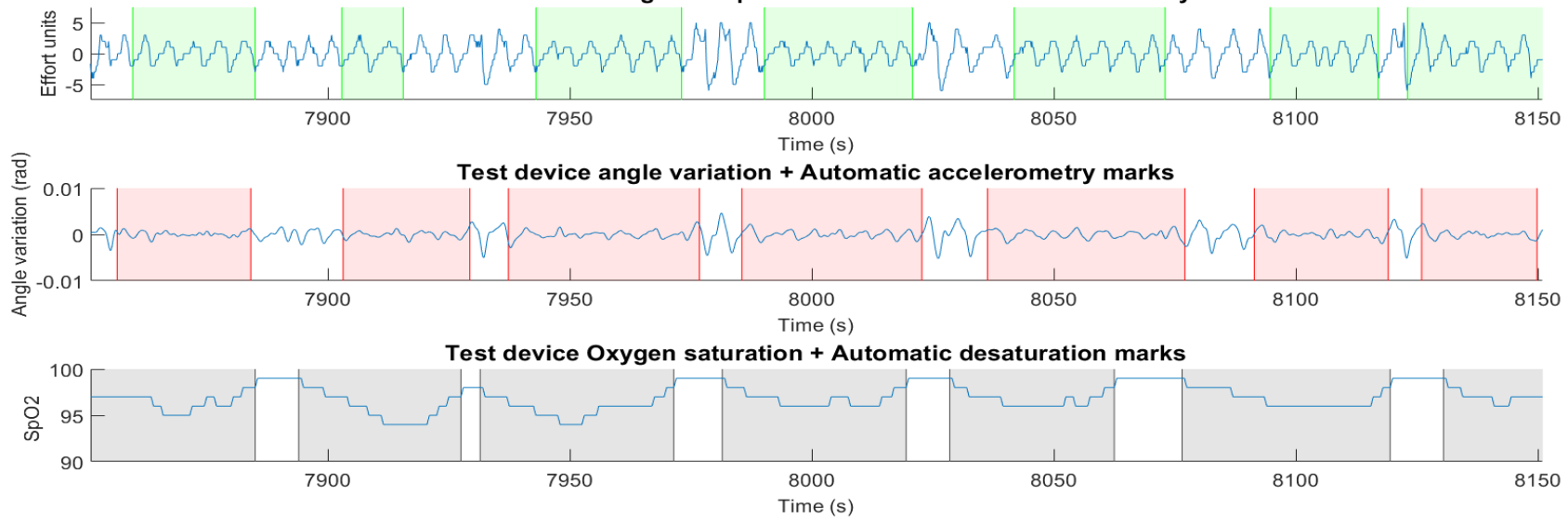

Figure 3. Reference device events compared to smartphone events 
Moreover, the performance of the automatic event detector has found a good event agreement, seen in Fig. 3, since the events of the airflow signal and chest effort of the reference system match with the events detected automatically on the angular signal and pulse oximetry of the automatic detector.

\section{DISCUSSION}

In this study we propose an automatic method to detect events linked to thoracic movement. Gold standard method for sleep assessment (PSG) could not be used as reference test since acquisitions were performed at home. Therefore, a commercial device for diagnosis of OSA at home was used.

In the first place, detection of subject movements (Fig. 1) is important because they generate too much acceleration and hide information of interest related to thoracic effort. Also, movements are usually followed by a change of the baseline. Since the magnitude of this change is greater than the oscillation produced by the thorax when breathing, if signal is not filtered separately on each side of position changes, a filter-derived ripple appears and modifies the signal of interest, affecting the calculation of the thoracic effort angle. We used subject position to establish starting and ending moments of the acquisition by determining when the subject was standing or laying in the bed in different positions.

In addition, the steps followed by the automatic detector to obtain the events (Fig. 2) succeeded in detecting those regions with a reduction of the oscillation angle and discarding possible false positive events. Since this method is based on the direction of the accelerometry vector, the variation of the magnitude of the vector was not considered. Nevertheless, as it can be seen in Fig. 3, angle oscillation reduction matched the reference device thoracic band and flow reduction. This proves that accelerometry can perform well to detect respiratory events including both apneas and hypopneas. We also expect to be able to differentiate between central and obstructive events, since central events should not produce any angle variation and angle envelope amplitude value should be close to zero. Even though, further study is required to validate this hypothesis.

Regarding the performance of the automatic detector, results from Table I show that overall sensitivity is slightly better in the automatic event detector method, while PPV is not, even though performance of the automatic method is still good. The main difference can be seen on the PPV values, which are better in the manual annotation. One explanation for this difference is that events detected manually by the doctors on the accelerometry signals were longer than automatically detected events. This increased the probability that an event detected by a doctor matched an event from the reference system, increasing then the PPV. Another possibility could be that medical expertise and event annotation experience in sleep assessment field plays a key role in determining whether an event has happened or not. This could influence the event occurrence decision and be more conservative to detect an event. Both hypotheses can be reinforced when results from Table I are combined with results from Table II. Medical manual annotation tends to underestimate AHI scores, which would support that either doctors were more conservative to annotate an event or that events detected were longer than usual and spanning more than 1 event of the reference.

Furthermore, oxygen saturation is another important aspect to consider when analyzing Se and PPV of the test system. In this study, each system used its own pulse oximeter and events from both systems have been detected related to desaturation episodes from their pulse oximeter. According to this setup, differences in pulse oximetry dynamics could influence the appearance of desaturation episodes and affect the detection of events. Differences in oximetry desaturation could produce over/under detection of events and reduce Se and PPV from the test device, influencing both manual and automatic event detection from the test device.

Finally, AHI estimation results from Table II show that the automatic method performed very well, achieving scores with almost the same index value as the reference.

\section{CONCLUSION}

In this study we explored the potential of accelerometry to obtain a respiratory signal related to thoracic movement and detect OSA related events. Since this has been a pilot study, results obtained from automatic algorithms developed to detect events are considered satisfactory. Nevertheless, further work is still needed to improve automatic detection and compare their results with manual events from a big database.

Smartphones appear to be promising systems to include automatic event detectors for improving diagnosis and monitoring of OSA patients at home. Accelerometry signal may provide powerful information to facilitate the event detection and help reach that goal.

\section{REFERENCES}

[1] P.E. Peppard, T. Young, J.H. Barnet, M. Palta, E.W. Hagen and K.M. Hla, "Increased prevalence of sleep-disordered breathing in adults". Am J Epidemiol, vol. 177, pp. 1006-14, May 2013

[2] R.N. Aurora, N.A. Collop, O. Jacobowitz, S.M. Thomas, S.F. Quan, and A.J. Aronsky, "Quality measures for the care of adult patients with obstructive sleep apnea.," J. Clin. Sleep Med. Off. Publ. Am. Acad. Sleep Med., vol. 11, no. 3, pp. 357-83, Mar 2015

[3] P.D. Hung, S. Bonnet, R. Guillemaud, E. Castelli, and P.T.N. Yen, "Estimation of respiratory waveform using an accelerometer," Proc. IEEE Int. Symp. Biomed. Imaging: From Nano to Macro, pp. 14931496, 2008.

[4] A. Bates, M.J. Ling, J. Mann, and D.K. Arvind, "Respiratory rate and flow waveform estimation from tri-axial accelerometer data," in Proc. Int. Conf. Body Sensor Netw., Singapore, pp. 144- 50, Jun 2010

[5] L. Estrada, A. Torres, L. Sarlabous, and R. Jané, "Respiratory signal derived from the smartphone built-in accelerometer during a Respiratory Load Protocol," in Proc. 37th Annu. Int. Conf. IEEE EMBS, pp. 6768-6771, 2015

[6] E. Fino and M. Mazzetti, "Monitoring healthy and disturbed sleep through smartphone applications: a review of experimental evidence", Sleep Breath, pp 1-12, Apr 2018

[7] H. Nakano et al., "Monitoring sound to quantify snoring and sleep apnea severity using a smartphone: Proof of concept," J Clin Sleep Med, vol. 10, no. 1, pp. 73-78, Jan. 2014

[8] J. Behar et al., "SleepAp: an automated obstructive sleep apnoea screening application for smartphones", IEEE J Biomed Health, vol. 19, no. 1, pp. 325-331, 2015

[9] M.A. Cámara, Y. Castillo, D. Blanco-Almazán, L. Estrada and R. Jané "mHealth tools for monitoring obstructive sleep apnea patients at home: proof-of-concept", Conf Proc IEEE Eng Med Biol Soc, pp. 1555-1558, Jul 2017 